\title{
HbA1c: More than just a number
}

CPD

Mawson Wang, Tien-Ming Hng

\section{Background}

Glycated haemoglobin, or $\mathrm{HbA1c}$, is the main biomarker used to assess long-term glycaemic control in individuals with diabetes, and it correlates with the development of complications.

\section{Objective}

The aim of this article is to provide an overview of $\mathrm{HbA} 1 \mathrm{c}$ to understand its role in the treatment of individuals living with diabetes. Topics discussed include recommended treatment targets, methods of measurement, causes of measurement inaccuracy and alternative means available to assess glycaemic control.

\section{Discussion}

HbA1c should not be interpreted in isolation; the measurement accuracy and other parameters, including treatment goals and comorbidities, need to be considered.
THE USE OF GLYCATED HAEMOGLOBIN, or HbA1c, has become the standard of assessing glycaemic control in patients with diabetes since the American Diabetes Association (ADA) recommended its use in $1988 .{ }^{1}$ For decades, the diagnosis of diabetes relied on glucose criteria through using either fasting glucose, random glucose or the $75 \mathrm{~g}$ oral glucose tolerance test (OGTT). HbA1c was initially not endorsed for the diagnosis of diabetes; however, assay improvements led to the ADA validating its use in 2010 as a diagnostic criterion for diabetes at a cutoff of $\geq 6.5 \%$, pre-diabetes between $5.7 \%$ and $6.4 \%$, and normal $<5.7 \%$. $^{2}$ The HbA1c test is listed on the Medicare Benefits Schedule (MBS) for subsidy once every 12 months for the diagnosis of diabetes (HbA1c $\geq 48 \mathrm{mmol} / \mathrm{mol}$ [ $\geq 6.5 \%]$ ) in high-risk individuals, and up to four times per year for monitoring of established diabetes. ${ }^{3}$

\section{What is HbA1c?}

Haemoglobin is the iron-containing oxygen-transport protein present in erythrocytes. Normal adult haemoglobin $(\mathrm{HbA})$ comprises a haem moiety and two globin chains, the $\alpha$ and $\beta$ chains ( $\alpha 2 \beta 2$ ), making up approximately 97\% of adult haemoglobin. ${ }^{4,5}$ Within $\mathrm{HbA}$, approximately $6 \%$ is glycated, of which the main component is $\mathrm{HbA} 1 \mathrm{c}(5 \%)$, with minor components of $\mathrm{HbA} 1 \mathrm{a}$ and $\mathrm{HbA1b}(1 \%) .{ }^{4} \mathrm{HbA} 1 \mathrm{c}$ results from the covalent attachment of glucose to the $\mathrm{N}$-terminal valine of the haemoglobin $\beta$-chain in a nonenzymatic process known as glycation. ${ }^{6}$

$\mathrm{HbA} 1 \mathrm{c}$ is dependent on the interaction between the concentration of blood glucose and the lifespan of the erythrocyte. ${ }^{4}$ As the mean erythrocyte lifespan is approximately 120 days, HbA1c acts as a surrogate marker of glucose concentration during the preceding 8-12 weeks. ${ }^{6}$ As a result of the continuous turnover of erythrocytes, it is estimated that only $50 \%$ of an HbA1c value represents glucose exposure in the preceding 30 days, while $40 \%$ represents exposure in the previous 31-90 days and $10 \%$ in the previous $91-120$ days. ${ }^{4}$

\footnotetext{
HbA1c in the diagnosis of diabetes $\mathrm{HbA} 1 \mathrm{c} \geq 48 \mathrm{mmol} / \mathrm{mol}(\geq 6.5 \%)$ in a laboratory using a validated method is diagnostic of diabetes. It should be measured in individuals at risk of diabetes. Advantages of measuring $\mathrm{HbA} 1 \mathrm{c}$ for this purpose include its convenience without a need for pre-test preparation, sample stability when collected and less day-to-day variability. However, it is limited by various conditions affecting its accuracy (Table 1), greater cost and low sensitivity. ${ }^{2}$
} 


\section{HbA1c targets}

The Diabetes Chronic Complications Trial (DCCT) and United Kingdom Prospective Diabetes Study (UKPDS) were two landmark trials that unequivocally demonstrated the benefit of glycaemic control for delaying and reducing the rate of end-organ complications in type 1 and type 2 diabetes, respectively. ${ }^{7,8}$ The DCCT showed that intensive insulin therapy leading to $\mathrm{HbA} 1 \mathrm{c}$ of $53 \mathrm{mmol} / \mathrm{mol}$ (7.0\%, in comparison to $77 \mathrm{mmol} / \mathrm{mol}$ [9.2\%] in the conventional arm) reduced the risk of retinopathy, nephropathy and neuropathy by $35-70 \% .{ }^{8}$ However, the risk of severe hypoglycaemia increased with lower HbA1c values. The UKPDS demonstrated that achieving an HbA1c of $53 \mathrm{mmol} / \mathrm{mol}$ (7.0\%) with intensive therapy using insulin or sulfonylurea (in comparison to $63 \mathrm{mmol} / \mathrm{mol}$ [7.9\%] in the conventional arm) led to a $25 \%$ risk reduction in microvascular, but not macrovascular, outcomes. ${ }^{7}$

Tight glycaemic control appears to provide greater benefit if implemented early in the disease process when compared with implementation at a more advanced disease stage. Microvascular rather than macrovascular events are reduced with tight glycaemic control in more established disease. ${ }^{9}$ Higher rates of hypoglycaemia were evident in trial arms with intensive glycaemic control, and particular caution should be exercised in older patients, those with near-normal glycaemic control or those with a higher risk of cardiovascular disease. ${ }^{9}$ Thus, while achieving HbA1c targets should form the focus of diabetes management, this needs to be weighed against the risks of intensive therapy. HbA1c targets should be individualised for each patient on the basis of their type of diabetes, life expectancy, risk of hypoglycaemia, duration of disease and other comorbidities. Guidance on HbA1c targets is detailed in the Australian Diabetes Society (ADS) recommendations (Table 2).

\section{Measurement of $\mathrm{HbA1c}$}

HbA1c assays work by separating glycated and non-glycated forms of haemoglobin, either on the basis of differences in the isoelectric point or by structure. ${ }^{6,10}$ There are two main methods of reporting HbA1c. The National Glycohaemoglobin Standardisation Program (NGSP) method reports values in $\%$, whereas the International Federation of Clinical Chemistry (IFCC) reports in $\mathrm{mmol} / \mathrm{mol} .{ }^{11}$ The latter was developed to standardise international reporting of $\mathrm{HbA1c}$. In Australia, both methods are reported concurrently. A conversion equation is as follows: NGSP $(\%)=0.0915 \times$ IFCC $(\mathrm{mmol} / \mathrm{mol})$ $+2.15 .{ }^{11}$ There is a small margin of error in which the HbA1c measurement may vary for the same sample. Most methods report variations in $\mathrm{HbA} 1 \mathrm{c}$ of $0.2-0.3 \%$, but others may be higher. ${ }^{12}$

Additionally, there is increasing use of point-of-care testing devices that quantify $\mathrm{HbA} 1 \mathrm{c}$ on the basis of structural differences and offer immediate turnaround of $\mathrm{HbA} 1 \mathrm{c}$ results during a single visit. All approved devices must meet NSGP criteria for analytical performance, and studies have shown comparable accuracy with laboratory values. ${ }^{13,14}$ Point-of-care $\mathrm{HbA} 1 \mathrm{c}$ tests will be listed on MBS from 1 November 2021. ${ }^{15}$

\section{Causes of falsely low or high HbA1c}

There are numerous causes of falsely low or high HbA1c measurements. These can be divided into factors that alter the lifespan or turnover of the erythrocyte, changes in glycation and haemoglobin, and assay-related artefacts (Table 1).

Conditions that reduce the erythrocyte lifespan, or increase its turnover so that there is shorter exposure to glucose, will lower HbA1c. Common causes include blood loss, haemolytic anaemia and hypersplenism. ${ }^{5} \mathrm{HbA} 1 \mathrm{c}$ may not be a reliable indicator of glycaemic control during pregnancy as there is reduced erythrocyte lifespan from 120 days to 90 days, and increased erythropoietin production. ${ }^{5} \mathrm{HbA} 1 \mathrm{c}$ values typically decline by $12-16$ weeks of gestation, plateau by 20-24 weeks and rise again in the third trimester. ${ }^{5}$ End-stage renal failure is also associated with falsely low $\mathrm{HbA} 1 \mathrm{c}$ levels due to chronic renal anaemia and reduced erythrocyte survival. Cystic fibrosis-related diabetes is associated with increased erythrocyte turnover and similarly causes low HbA1c. ${ }^{16}$ Conversely, conditions that prolong the erythrocyte lifespan or reduce its turnover result in higher HbA1c. Common conditions include iron, vitamin B12 and folate deficiency anaemias and asplenia. ${ }^{5}$ Erythropoietin-stimulating agents and iron infusions in patients with renal failure can lead to falls in $\mathrm{HbA} 1 \mathrm{c}$, likely due to formation of new erythrocytes with reduced exposure to glucose and hence glycation. ${ }^{17}$

Haemoglobinopathies are genetic defects that result in abnormal structure of the globin chains, which in turn result in variable effects on $\mathrm{HbA} 1 \mathrm{c}$ depending on the method of measurement and assay. ${ }^{5}$ This is similarly the case for haemoglobin variants. Vitamin $\mathrm{E}$ at doses of 600-1200 mg daily may reduce protein glycation, hence reducing $\mathrm{HbA} 1 \mathrm{c} .{ }^{5}$ Blood transfusions can increase the rate of glycation in red blood cells stored in high glucose concentrations for up to 42 days. ${ }^{18}$ However, when patients are transfused with red blood cells from a non-diabetic patient, increased erythrocyte turnover and a dilutional effect may falsely lower HbA1c. ${ }^{5}$

Numerous assay-related artefacts can affect the HbA1c. Endogenous interference from hypertriglyceridaemia (>19.7 mmol/L) and hyperbilirubinaemia (>342 $\mu \mathrm{mol} / \mathrm{L}$ ), and detection of carbamylhaemoglobin in patients with uraemia can all falsely elevate HbA1c. ${ }^{5}$

Finally, ethnic differences have been noted. Non-Caucasian populations have higher HbA1c values than Caucasian populations even after adjustment for confounders such as socioeconomic status, obesity and other diabetic factors. ${ }^{19}$ Non-glycaemic genetic determinants of glycation and differences in erythrocyte survival are potential causes. ${ }^{19}$

\section{Is HbA1c the best method of assessing glycaemic control and are there alternatives?}

Studies have indicated that progression of diabetic complications cannot be solely explained by HbA1c, as complications may occur despite lower-than-average 
Table 1. Causes of falsely low or high glycated haemoglobin (HbA1c) ${ }^{10,5,27}$

\begin{tabular}{lll}
\hline Mechanism & Falsely low HbA1c & Falsely high HbA1c \\
\hline Change in red blood cell & Acute and chronic blood loss & Iron deficiency \\
lifespan or turnover & Renal failure & Vitamin B12 deficiency \\
& Haemolytic anaemia & Folate deficiency \\
& Spherocytosis & Asplenia \\
& Hypersplenism & \\
& Pregnancy & \\
Iron/erythropoietin- & \\
stimulating agent & \\
& administration & \\
& Blood transfusion & \\
Cystic fibrosis-related & \\
diabetes & \\
\hline Change in glycation & Vitamin E & Blood transfusion \\
\hline Altered haemoglobin & Haemoglobin variants & \\
& Haemoglobinopathies & \\
\hline Assay-related artifacts & & Hypertriglyceridaemia \\
& & Hyperbilirubinaemia \\
& & Araemia \\
& & Aspirin-induced acetylated \\
& & carboxyhaemoglobin \\
\hline
\end{tabular}

HbA1c, and vice versa. ${ }^{20}$ The most likely explanation is that $\mathrm{HbA} 1 \mathrm{c}$ does not account for day-to-day glycaemic variabilities. Patients with widely differing glucose profiles may have the same HbA1c (Figure 1), and the use of HbA1c alone without any corroborative glucose measurements will not allow appreciation of intra-day glycaemic excursions. A reduction in glycaemic variability alone - for example, by hypoglycaemic avoidance - can lead to improved quality of life. ${ }^{21}$

There are alternative methods of assessing glycaemic control. Self-monitoring of blood glucose (SMBG) provides an indication of day-to-day variability. Average glucose levels may be extrapolated from the HbA1c using the formula: average blood glucose level $(\mathrm{mmol} / \mathrm{L})=2 \times \mathrm{HbA} 1 \mathrm{c}(\%)-6 \mathrm{mmol} / \mathrm{L}^{10}$ However, infrequent SMBG will not provide glucose trends nor diagnose nocturnal or asymptomatic hypoglycaemia. ${ }^{22}$

Continuous glucose monitoring (CGM) using either a flash or continuous system is a newer method of measuring interstitial glucose measurements at five-minute intervals. Whereas HbA1c can only provide information on long-term control, CGM offers comprehensive information on glucose variability and trends, thus providing clinicians with the ability to individualise diabetes management depending on the glycaemic pattern. ${ }^{22}$ The CGM report also includes the 'glucose management indicator', which is an estimated HbA1c measurement that is derived from mean glucose levels and thus not directly comparable to the laboratory-measured HbA1c. ${ }^{22}$ Other useful parameters obtained from CGM include the 'time in range' and the glucose 'coefficient of variation'. ${ }^{22}$ As CGM becomes more widely accessible and used in outcome trials, it will likely become increasingly important as a tool for assessing glycaemic control. ${ }^{23}$

Fructosamine is an alternative marker of glucose levels, as it is the product of glycation between glucose and protein, predominantly albumin. ${ }^{5}$ Glycated albumin is an example of a fructosamine. As the half-life of albumin ( 20 days) is much shorter than that of erythrocytes, it reflects glycaemic control over the past 2-3 weeks. ${ }^{5}$ Nevertheless, fructosamine and glycated albumin are also subject to falsely low readings in states of hypoproteinaemia or hypoalbuminaemia. ${ }^{5}$ These tests may be considered when conditions affecting erythrocytes may affect $\mathrm{HbA} 1 \mathrm{c}$; however, their prognostic significance is unclear and thus not endorsed in official guidelines. ${ }^{17,24}$

\section{Glucose monitoring in specific populations All populations}

If the HbA1c measurement is deemed to be inaccurate (Table 1), assessment of glycaemic control should rely on SMBG (or CGM). To reduce glucose variability, normalisation of fasting and postprandial blood glucose levels should be strived for even if the target HbA1c is met, but this needs to be balanced against the burden of additional medications and their side-effect profile.

\section{Type 1 diabetes}

Apart from HbA1c and SMBG, CGM is particularly useful in patients with type 1 diabetes and may decrease time spent in hypoglycaemia. ${ }^{25}$ Under the National Diabetes Services Scheme, CGM is currently subsidised for children and young adults aged under 21 years, adults aged 21 years and over with valid concessional status, and women who are actively planning pregnancy, pregnant or immediately postpartum.

\section{Renal failure}

Fructosamine and glycated albumin can be used as alternative glycaemic markers to HbA1c; however, low protein and albumin states limit their usage. Assessment of an SMBG diary over a period of time is likely to be more useful; CGM could also be considered. ${ }^{26}$

\section{Pregnancy}

A $75 \mathrm{~g}$ OGTT rather than a HbA1c test should be used to diagnose diabetes. ${ }^{5}$ SMBG should be used for glucose 
monitoring and medication adjustment. CGM is another option, particularly for individuals with type 1 diabetes.

\section{Patients with an unexplained discrepancy between HbA1c and glucose readings}

It is important to first ensure appropriate SMBG technique and exclude hardware issues with the glucometer. Accuracy of glucometer measurements can be assessed using high and low control solutions from the manufacturer. Assessment of $\mathrm{HbA1c}$ using a different laboratory or assay may also be considered to confirm the accuracy of the initial measurement. If the discrepancy remains, frequent SMBG or CGM can be used to investigate this further.

\section{Conclusion}

$\mathrm{HbA} 1 \mathrm{c}$ is a widely ordered and reviewed test in general practice. It is useful as an adjunct to other parameters such as SMBG or CGM to provide a holistic understanding of an individual's recent glycaemic control. Care must be taken to consider various conditions and scenarios that may affect its measurement.

\section{Key points}

Table 2. Australian Diabetes Society position statement on recommended glycated haemoglobin (HbA1c) targets $^{9}$

\begin{tabular}{ll}
\hline Condition & HbA1c target \\
\hline General target & $\leq 53 \mathrm{mmol} / \mathrm{mol}(7.0 \%)$
\end{tabular}

Diabetes of short duration and no clinical

cardiovascular disease

\begin{tabular}{ll}
\hline Lifestyle modifications \pm metformin & $\leq 42 \mathrm{mmol} / \mathrm{mol}(6.0 \%)$ \\
\hline $\begin{array}{l}\text { Requiring any antidiabetic agent other than metformin } \\
\text { or insulin }\end{array}$ & $\leq 48 \mathrm{mmol} / \mathrm{mol}(6.5 \%)$ \\
\hline Requiring insulin & $\leq 53 \mathrm{mmol} / \mathrm{mol}(7.0 \%)$ \\
\hline Pregnancy or preconception & $\leq 42 \mathrm{mmol} / \mathrm{mol}(6.0 \%)$ \\
\hline Diabetes of longer duration or clinical cardiovascular disease & $\leq 53 \mathrm{mmol} / \mathrm{mol}(7.0 \%)$ \\
\hline Severe hypoglycaemia or hypoglycaemia unawareness & $\leq 64 \mathrm{mmol} / \mathrm{mol}(8.0 \%)$ \\
\hline Major comorbidities likely to limit life expectancy & $\begin{array}{l}\text { Symptomatic treatment of } \\
\text { hyperglycaemia, aim for blood } \\
\text { glucose level <15 mmol/L }\end{array}$ \\
\hline
\end{tabular}

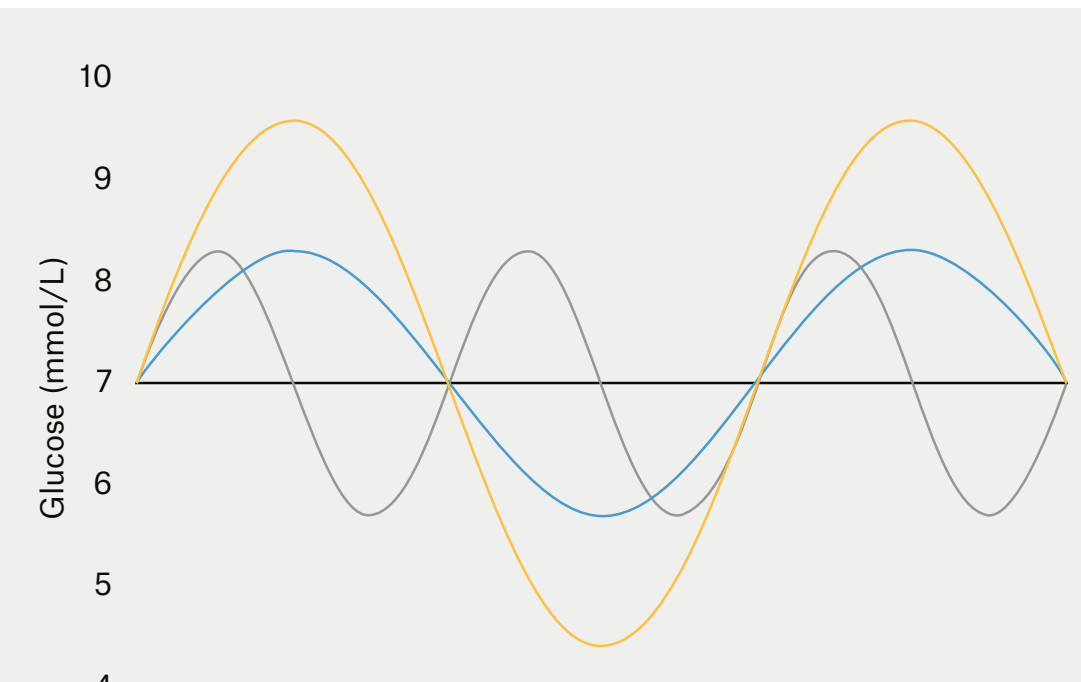

4

Time

Figure 1. An example of three glucose profiles exhibiting differing degrees of glucose variability with the same mean blood glucose and glycated haemoglobin

- HbA1c is the most widely accepted marker of glycaemic control in individuals with diabetes, and it correlates with risk of diabetic complications.

- HbA1c targets should be discussed and individualised according to type and duration of diabetes, life expectancy, risk of hypoglycaemia and other comorbidities.

- Numerous factors may falsely elevate or lower $\mathrm{HbA1c}$, including anaemia, iron deficiency, renal failure and pregnancy.

- HbA1c does not take into account glucose variability, and two individuals with the same $\mathrm{HbA} 1 \mathrm{c}$ may exhibit vastly different glucose profiles.

- Alternative methods of assessing glycaemic control, such as SMBG or CGM, can provide additional evidence about glycaemic excursions and should be used in conjunction with $\mathrm{HbA} 1 \mathrm{c}$ if the data are available for review.

\section{Authors}

Mawson Wang MD, Endocrinology Advanced Trainee, Department of Diabetes and Endocrinology, Blacktown-Mt Druitt Hospital, Sydney, NSW; Blacktown Clinical School, School of Medicine, Western Sydney University, Sydney, NSW

Tien-Ming Hng MBBS, PhD, FRACP, Head of Endocrinology, Department of Diabetes and Endocrinology, Blacktown-Mt Druitt Hospital, Sydney, NSW; Blacktown Clinical School, School of Medicine, Western Sydney University, Sydney, NSW

Competing interests: None.

Funding: None.

Provenance and peer review: Not commissioned, externally peer reviewed.

Correspondence to:

mawson.wang@health.nsw.gov.au

\section{References}

1. Standards of medical care for patients with diabetes mellitus. Diabetes Care 1989:12(5):365-68. doi: 10.2337/diacare.12.5.365

2. American Diabetes Association. 2. Classification and diagnosis of diabetes: Standards of medical 
care in diabetes - 2021. Diabetes Care 2021;44 Suppl 1:S15-33. doi: 10.2337/dc21-S002.

3. Australian Government Department of Health. Medicare Benefits Schedule. Canberra, ACT: MBS Online, 2020. Available at www.mbsonline.gov. $\mathrm{au} /$ internet/mbsonline/publishing.nsf/Content/ Home [Accessed 14 January 2021].

4. Lenters-Westra E, Schindhelm RK, Bilo HJ, Slingerland RJ. Haemoglobin A1c: Historical overview and current concepts. Diabetes Res Clin Pract 2013;99(2):75-84. doi: 10.1016/j. diabres.2012.10.007.

5. Radin MS. Pitfalls in hemoglobin A1c measurement: When results may be misleading. J Gen Intern Med 2014;29(2):388-94. doi: 10.1007/s11606-013-2595-x.

6. Little RR, Sacks DB. HbA1c: How do we measure it and what does it mean? Curr Opin Endocrinol Diabetes Obes 2009;16(2):113-18. doi: 10.1097/MED.0b013e328327728d.

7. Turner R. Intensive blood-glucose control with sulphonylureas or insulin compared with conventional treatment and risk of complications in patients with type 2 diabetes (UKPDS 33). UK Prospective Diabetes Study (UKPDS) Group. Lancet 1998;352(9131):837-53. Erratum in: Lancet 1999;354(9178):602.

8. Diabetes Control and Complications Trial Research Group, Nathan DM, Genuth S, et al. The effect of intensive treatment of diabetes on the development and progression of long-term complications in insulin-dependent diabetes mellitus. N Engl J Med 1993;329(14):977-86. doi: 10.1056/NEJM199309303291401.

9. Cheung NW, Conn JJ, d'Emden MC, et al. Position statement of the Australian Diabetes Society: Individualisation of glycated haemoglobin targets for adults with diabetes mellitus. Med J Aust 2009;191(6):339-44. doi: 10.5694/j.1326-5377.2009.tb02819.x.

10. Phillips PJ. HbA1c and monitoring glycaemia. Aust Fam Physician 2012;41(1-2):37-40.

11. Little RR, Rohlfing CL. HbA 1c standardization: Background, progress and current issues. Lab Med 2009;40(6):368-73. doi: 10.1309/LM3DUSEIBXHTVZ70.

12. Heinemann L, Freckmann G. Quality of HbA1c measurement in the practice: The German perspective. J Diabetes Sci Technol 2015;9(3):687-95. doi: 10.1177/1932296815572254.

13. Szablowski CJ, Suscha E, Davis K, et al. Pointof-care $\mathrm{HbA1c}$ - A case for diabetes screening and diagnosis. Diabetes 2018;67 Suppl 1:1518-P. doi: 10.2337/db18-1518-P.

14. Whitley HP, Yong EV, Rasinen C. Selecting an A1C point-of-care instrument. Diabetes Spectr 2015;28(3):201-08. doi: 10.2337/diaspect.28.3.201.

15. Australian Government Department of Health. \$2.1 million to support Australians with diabetes. Canberra, ACT: Commonwealth of Australia. Available at www.health.gov.au/ ministers/the-hon-greg-hunt-mp/media/21million-to-support-australians-with-diabetes [Accessed 17 May 2021].

16. Hardin DS, Grilley K, Baron B, Hale KA. Accelerated red blood cell turnover can invalidate the use of hemoglobin A1c as a diagnostic test for cystic fibrosis related diabetes. Pediatr Res 1999;45(4):90. doi: 10.1203/00006450199904020-00537.

17. Ng JM, Cooke M, Bhandari S, Atkin SL, Kilpatrick ES. The effect of iron and erythropoietin treatment on the $\mathrm{A} 1 \mathrm{C}$ of patients with diabetes and chronic kidney disease. Diabetes Care 2010;33(11):2310-13. doi: 10.2337/dc10-0917.
18. Prosenz J, Öhlinger T, Müllner EW, et al. Glycated hemoglobin concentrations of red blood cells minimally increase during storage under standard blood banking conditions. Transfusion 2019;59(2):454-57. doi: 10.1111/trf.14956.

19. Herman WH, Cohen RM. Racial and ethnic differences in the relationship between $\mathrm{HbA1c}$ and blood glucose: Implications for the diagnosis of diabetes. J Clin Endocrinol Metab 2012;97(4):1067-72. doi: 10.1210/jc.2011-1894.

20. The relationship of glycemic exposure (HbA1c) to the risk of development and progression of retinopathy in the diabetes control and complications trial. Diabetes 1995;44(8):968-83.

21. Green AJ, Fox KM, Grandy S; SHIELD Study Group. Self-reported hypoglycemia and impact on quality of life and depression among adults with type 2 diabetes mellitus. Diabetes Res Clin Pract 2012;96(3):313-18.

22. Danne T, Nimri R, Battelino T, et al. International consensus on use of continuous glucose monitoring. Diabetes Care 2017;40(12):1631-40. doi: 10.2337/dc17-1600.

23. Klein KR, Buse JB. The trials and tribulations of determining $\mathrm{HbA1c}$ targets for diabetes mellitus. Nat Rev Endocrinol 2020;16(12):717-30. doi: 10.1038/s41574-020-00425-6.

24. American Diabetes Association. 6. Glycemic targets: Standards of medical care in diabetes-2019. Diabetes Care 2019;42 Suppl 1:S61-70. doi: 10.2337/dc19-S006.

25. Mauras N, Fox L, Englert K, Beck RW. Continuous glucose monitoring in type 1 diabetes. Endocrine 2013;43(1):41-50. doi: 10.1007/s12020-012-9765-1.

26. Tuttle KR, Bakris GL, Bilous RW, et al. Diabetic kidney disease: A report from an ADA Consensus Conference. Diabetes Care 2014;37(10):2864-83. doi: 10.2337/dc14-1296.

27. Homa K, Majkowska L. Difficulties in interpreting $\mathrm{HbA}(1 \mathrm{c})$ results. Pol Arch Med Wewn 2010;120(4):148-54. 